\title{
The Use of Double- Needle Canula Method for Temporomandibular Joint Arthrocentesis: Clinical Report
}

\author{
Alper Alkana, DDS, PhD
}

Burcu Baş ${ }^{b}$ DDS, PhD

\section{ABSTRACT}

Objective: The purpose of this study is to demonstrate a temporomandibular lavage instrument with double needles in a single canula that make the procedure easier for surgeons.

Materials and methods: 38 year old woman was referred to our department with pain on the right temporomandibular joint (TMJ) region and restricted mouth opening. Magnetic resonance imaging reveals anterior disc displacement without reduction of the right TMJ. TMJ lysis and lavage was performed with double needle canula method.

Results: The upper joint space was successfully lavaged with $50 \mathrm{~mL}$ of $0.9 \%$ saline solution. Maximal mouth opening and lateral jaw movement increased and jaw functions improved immediately after the procedure.

Conclusion: It is recommended as a simple alternative to classical arthrocentesis with two needles that it is easy to use and enables to perform lysis and lavage with a single puncture. (Eur J Dent 2007;1:179-182)

Key Words: Arthrocentesis; TMD; TMJ lavage.

\section{INTRODUCTION}

The technique of temporomandibular joint (TMJ) arthrocentesis and lavage described by Nitzan et $\mathrm{al}^{1}$ is a simple means of releasing the "stuck" disc from the fossa by irrigation of the superior joint space under local anesthesia on an

a Department of Oral and Maxillofacial Surgery, Erciyes University, Faculty of Dentistry, Kayseri, TURKEY

${ }^{b}$ Department of Oral and Maxillofacial Surgery, Ondokuz Mayıs University, Faculty of Dentistry, Samsun, TURKEY

- Corresponding Author: Dt. Burcu Bas Ondokuz Mayıs University, Faculty of Dentistry, Department of OMS, 55139, Kurupelit,

Samsun, TÜRKIYE

E-mail: burcubsdyahoo.com

Tel: +90 $3623121919-3480$ Fax: +90 3624576032 outpatient basis. Preliminary results of this procedure have been promising in effectively establishing increased range of motion, improved function and decreased pain. ${ }^{1,2}$

The technique of arthrocentesis reported in the literature itself varies considerably. A number of irrigating solutions have been used in varying quantities and at different pressures.3,4 The volume of solution used for TMJ lavage varied widely and ranged from 50 to $500 \mathrm{~mL} .{ }^{5}$ Kaneyama et $\mathrm{al}^{4}$ reported that $200 \mathrm{~mL}$ of perfusate was required to significantly decrease the concentration of protein in the joint cavity and only $50 \mathrm{~mL}$ was required for BK and IL-6. Whereas Zardeneta et $\mathrm{al}^{6}$ reported that approximately $100 \mathrm{~mL}$ of total perfusate is sufficient for therapeutic lavage. Arthrocenthesis can be performed either under low pressure using an elevated infusion bag or under sufficient 
pressure by using a syringe..$^{5}$ Outcomes of arthrocentesis are defined with great variations in the literature ranging from $\% 70$ to $\% 91$. Emshoff et al ${ }^{7}$ reported the success rate of arthrocentesis $53 \%$ in patients with anterior disc displacements without reduction and they stated that the efficiency and effectiveness of the procedure is also depend on clinical variables such as age, gender, time since pain onset, pain level, and mandibular range of motion.

Arthrocentesis is a relatively simple technique in office procedure which allows expansion of the joint space, lysis of adhesions and lavage via blind input and outflow catheters however surgeons can be faced with some clinical difficulties with two needles during the procedure such as displacement of the needles during the irrigation and difficulty of inserting the outflow needle to the right place. TMJ lavage instrument (ACE Surgical Supply, Inc., Brocton, MA) with double needle in a single canula has been introduced in the literature previously. ${ }^{8}$ However it was not in routine use

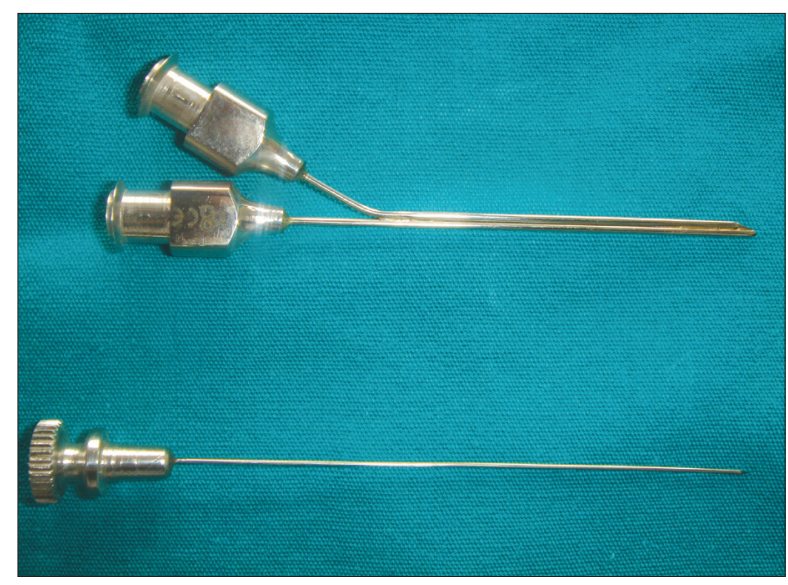

Figure 1. The view of the canula and a sharp-tipped throcar.

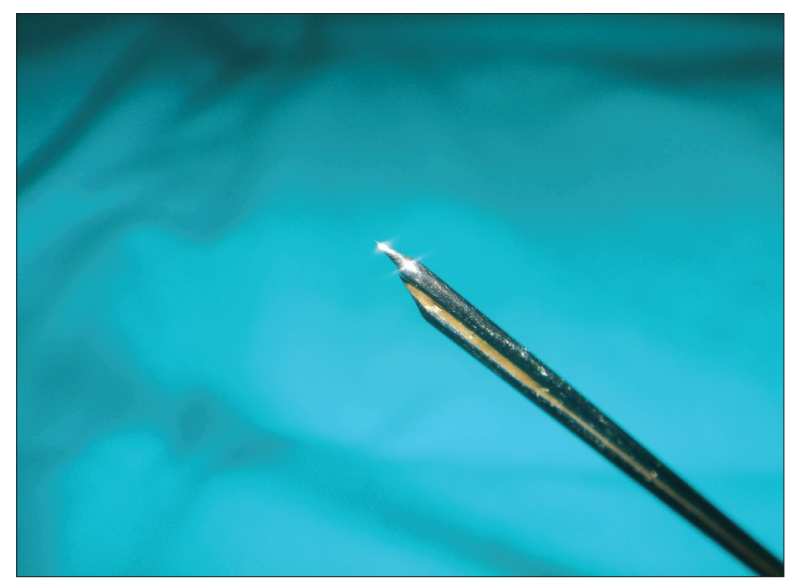

Figure 3. The view of the sharp-tipped throcar inserted into the irrigation tube for the enterance into joint space through skin. probably because of the lack of presentation. The purpose of this report is to describe this instrument and state the advantages of the technique.

\section{MATERIALS AND METHODS}

A 34 years old woman was referred to our clinic with pain in the right preauricular region and restricted mouth opening of 4 months' duration. Clinical examination revealed tenderness of the right TMJ region. Maximum mouth opening was $30 \mathrm{~mm}$ and there was a definite deflection of the mandibular midline to the right upon opening. The patient had a normal range of lateral movement to the right side $(9 \mathrm{~mm})$, but left lateral movement was limited to $5 \mathrm{~mm}$. She had a prior history of asymptomatic TMJ clicking, that disappeared with a sudden decrease in mouth opening. MRI showed anterior disc displacement without reduction on the right TMJ. We planned to perform arthocentesis with double needle canula method.

We re-designed the instrument as previously described in the literature ${ }^{8}$ and manufactured

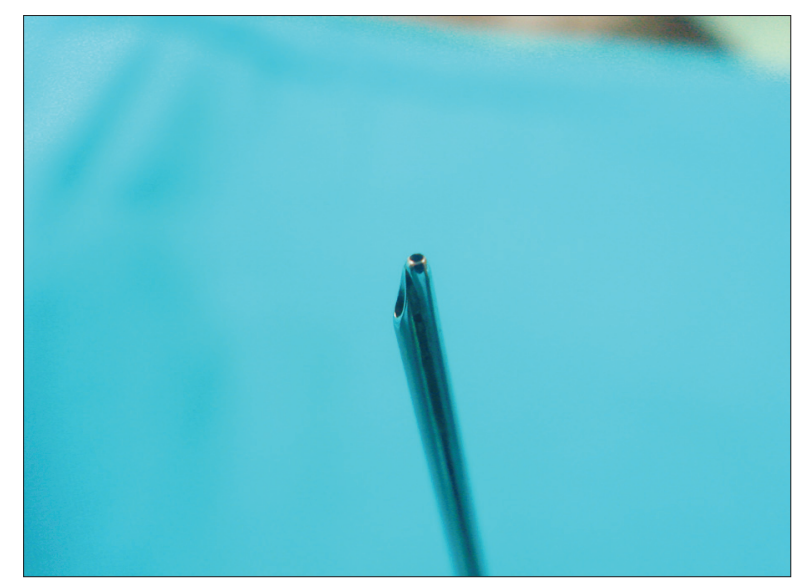

Figure 2. The view of the irrigation and aspiration tubes in the canula.

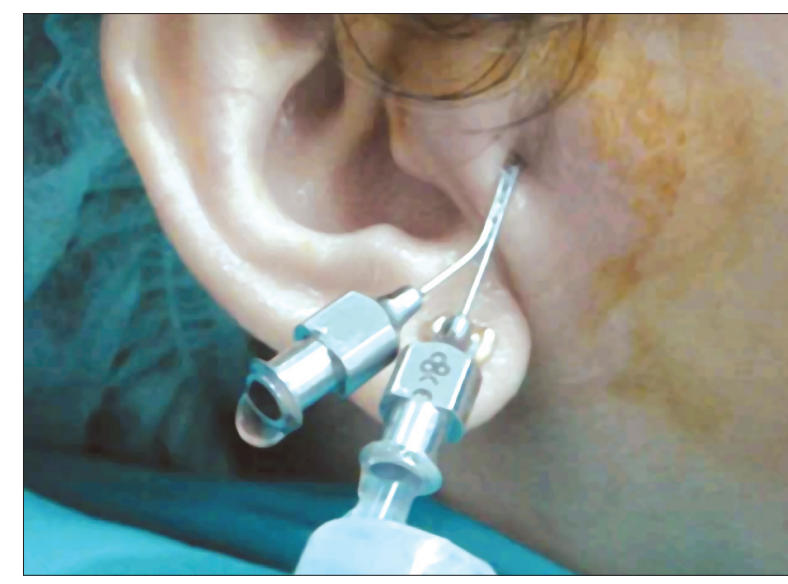

Figure 4. The view of clinical use of the instrument. 
in a local company (Bahadır Medical Technology, Samsun, Turkey). The instrument was made of stainless steel. It contains two adjacent irrigation and aspiration tubes in a single canula and a sharp-tipped throcar for entrance through the skin (Figure 1). The length of the canula is $80 \mathrm{~mm}$ and the diameters of the tubes in the canula are $1 \mathrm{~mm}$ and $0.5 \mathrm{~mm}$ (Figure 2). The diameter of the sharptipped throcar which is inserted into the irrigation tube is $0.8 \mathrm{~mm}$ (Figure 3).

After local anesthesia injection around the joint space, the canula with the sharp throcar was introduced into the superior compartment of the joint using the canthus-tragus line as a guide. The sharp throchar was then removed from irrigation tube, and a $10-\mathrm{mL}$ syringe containing salin solution connected to the instrument (Figure 4). $2 \mathrm{~mL}$ saline solution was injected to widen the upper joint space by closing the aspiration tube with a cover. Then the cover was taken and the joint is lavaged with $50 \mathrm{~mL}$ of $0.9 \%$ normal saline solution and then the canula was withdrawn. The patient was prescribed anti-inflammatory drug for the following 7 days. Occlusal appliance therapy was started again after procedure.

\section{RESULTS}

No complication was occurred during the procedure. Maximal mouth opening increased to 40 $\mathrm{mm}$ and left lateral jaw movement increased to 8 $\mathrm{mm}$ immediately after the procedure. At 3 month follow up clinical evaluation showed a significant reduction in TMJ pain and an increase in mandibular range of motion.

\section{DISCUSSION}

In the early 1990's, the success of simple arthroscopic lavage and lysis 9,10 for the management of closed lock led to the development of an even less invasive and much simpler technique of TMJ arthrocentesis which rapidly gained widespread acceptance as a popular treatment modality for the management of closed-lock of the TMJ.2,11 $\mathrm{Ar}$ throcentesis is recognized increasingly as first line surgical intervention in patients who do not respond to conservative management. In some cases with long-standing (chronic) closed lock are resistant to TMJ arthrocentesis and TMJ arthroscopic lavage and lysis must be used. It has led to a better understanding of the normal and abnormal relation- ships of the intra-articular disc and the associated internal derangements. ${ }^{12}$ However, arthroscopy is an equipment-dependent procedure that relies considerably on expensive and complex technology and it is difficult for many surgeons to develop this expertise. $^{13}$

TMJ arthrocentesis entails placing two needles into the superior joint space for the purposes of lysis and lavage via hydraulic distension. Ringer's lactate solution is injected through one needle and the second needle acts an outlet valve. The technique is performed on an outpatient basis under local anesthesia. ${ }^{14}$ Advantages of TMJ arthrocentesis are that it is a simple, inexpensive, and minimally invasive procedure with little morbidity that can easily be performed in an outpatient setting. ${ }^{11}$ However; it can be sometimes difficult for inexperienced surgeons to find the exact places for the needles. Besides, maintaining the exact place during the efficient lavage can also be a challenging procedure. Repetitive insertions of needles to find the right place can damage capsular tissues, increases the risk of facial nerve injury and traumatization of the capsule can also aggregate inflammation in TMJ.

In this report, TMJ lavage instrument with double needle in a single canula was successfully used in a patient with anterior disc displacement without reduction. The main advantage of the instrument is that efficient arthrocentesis can be achieved without inserting a second needle for outflow and there is no risk of losing the right place during the efficient lavage. Irrigation can be performed either with low pressure with an elevated infusion bag or with high pressure. The diameter of the canula is small enough to enter into the joint with local anesthesia. However, in our opinion the main limitation of the technique is that it can be more difficult to enter the narrow joint spaces with this instrument in severe degenerative joints with osteophytic changes. Arthrocenthesis with two needles should be preferred in such cases.

\section{CONCLUSIONS}

The double-needle canula method proved to be a feasible alternative for the performance of arthrocentesis of the TMJ. However, further researches are needed to compare the outcomes of this technique and classical arthrocenthesis. 


\section{REFERENCES}

1. Nitzan DW, Dolwick MF, Martinez GA. Temporomandibular joint arthrocentesis. A simplified treatment for severe limited mouth opening. J Oral Maxillofac Surg 1991;49:11631167.

2. Dimitroulis G, Dolwick MF, Martinez A. Temporomandibular joint arthrocentesis and layage for the treatment of closedlock: a follow-up study. Br J Oral Maxillofac Surg $1995 ; 33: 23-27$.

3. Yura S, Totsuka Y. Relationship between effectiveness of arthrocentesis under sufficient pressure and conditions of the temporomandibular joint. J Oral Maxillofac Surg 2005;63:225-228.

4. Kaneyama K, Segami N, Nishimura M, Sato J, Fujimura $\mathrm{K}$, Yoshimura $\mathrm{H}$. The ideal lavage volume for removing bradykinin, interleukin-6, and protein from the temporomandibular joint by arthrocentesis. J Oral Maxillofac Surg 2004;62:657-661.

5. Al-Belasy FA, Dolwick MF. Arthrocentesis for the treatment of temporomandibular joint closed lock: a review article. Int J Oral Maxillofac Surg 2007;18.

6. Zardeneta G, Milam SB, Schmitz JP. Elution of proteins by continuous temporomandibular joint arthrocentesis. J Oral Maxillofac Surg 1997;55:709-716.

7. Emshoff R. Clinical factors affecting the outcome of arthrocentesis and hydraulic distension of the temporomandibular joint. Oral Surg Oral Med Oral Pathol Oral Radiol Endod 2005; 100:409-414.

8. McCain JP. Principles and Practice of Temporomandibular Joint Arthroscopy. Mosby:1996.

9. Sanders B. Arthroscopic surgery of the temporomandibular joint: Treatment of internal derangement with persistent closed lock. Oral Surg Oral Med Oral Pathol 1986;62:361372.

10. Nitzan DW, Dolwick MF. Arthroscopic lavage and lysis of the temporomandibular joint: A change in perspective. $J$ Oral Maxillofac Surg 1990;48:798-801.

11. Dolwick MF. The role of temporomandibular joint surgery in the treatment of patients with internal derangement. Oral Surg Oral Med Oral Pathol Oral Radiol Endod 1997;83:150155.

12. Nitzan DW, Samson B, Better H. Long-term outcome of arthrocentesis for sudden-onset, persistent, severe closed lock of the temporomandibular joint. J Oral Maxillofac Surg 1997;55:151-157.

13. Indresano AT. Surgical arthroscopy as the preferred treatment for internal derangements of the temporomandibular joint. J Oral Maxillofac Surg 2001;59:308-312.
14. Frost DE, Kendell BD. The use of arthrocentesis for treatment of temporomandibular joint disorders. J Oral Maxillofac Surg 1999;57:583-587. 